\title{
Title
}

\section{Effects of altered parental folate and one-carbon nutrient status on offspring growth and metabolism}

\section{Author \\ Jimmy W. Crott PhD}

Jean Mayer USDA Human Nutrition Research Center on Aging at Tufts University

711 Washington St. Boston, MA

T: +1 6175563117

E: jimmy.crott@tufts.edu

\section{Key Words}

Folic acid, folate, vitamin $B_{12}$, maternal, paternal, offspring, insulin resistance, adiposity, peri-conceptional.

\section{Abbreviations}

DFE, Dietary folate equivalents; HOMA-IR, Homeostatic model assessment (of) insulin resistance; IR, insulin resistance; IUGR, intra uterine growth restriction; oGTT, oral glucose tolerance test; NHANES, National Health and Nutrition Examination Survey; RBC, red blood cell; SGA, small for gestational age;

\section{Acknowledgements}

This work was funded in part by the Agricultural Research Service of the United States Department of Agriculture, Cooperative Agreements \#58-1950-4-003. Opinions and ideas expressed in this publication are not necessarily those of the U.S. Department of Agriculture. The author reports no conflict of interest. 


\section{Introduction}

Since the publication of the 'thrifty phenotype' hypothesis (Hales and Barker 1992), the potential influence of a mother's nutritional status on her children's risk for disease has captured the imagination of epidemiologists and basic scientists alike. This landmark hypothesis arose from observations that geographical differences in death rates from cardiovascular disease across England and Wales were closely related to differences in infant mortality - most cases of which were related to low birth weight (Barker and Osmond 1986). Support for this hypothesis subsequently came from the findings of the Hertfordshire study in which men with the lowest weight at birth and 1 year of age had the highest rates of death from ischemic heart disease (Barker et al. 1989).

Although poor diet and low folate intake havebeen associated with an elevated risk for neural tube defects since the early 1980's (reviewed in (Lumley et al. 2001)), the realization that more subtle changes in offspring could also be induced by altering maternal one-carbon status and result in altered risk for disease later in life probably arose from the seminal findings of Wolff and Cooney. They demonstrated that the coat color of agouti $\left(A^{v y}\right)$ mouse offspring was sensitive to maternal diet; dams fed a methyl-supplemented diet (high choline, betaine, folic acid, vitamin $B_{12}$ ) were more likely to produce offspring with brown (psuedoagouti) coats while dams fed a control diet had offspring which were mostly yellow (Wolff et al. 1998). Importantly, despite having the same genotype, $A^{v y}$ mice with a yellow coat color become obese and display elevated circulating insulin and impaired glucose tolerance while mice with a brown coat color remain lean, normoinsulinemic and normoglycemic(Wolff et al. 1986).They later reported that the darker the coat color, the more methylated the responsible $A^{v y}$ allele was (Cooney et al. 2002). Demonstration in humans that maternal one-carbon status can affect offspring methylation comes from studies such as those in the Netherlands in which maternal folic acid supplementation was associated with a significant elevation in lgf2 methylation in their children (Steegers-Theunissen et al. 2009).

In this review I summarize the findings of human and mouse studies investigating the relationship between maternal one-carbon nutrient status and offspring growth, metabolism and obesity and have attempted to distill common themes from this highly heterogeneous body of literatureas well as identify areas ripe for future research. Although maternal one-carbon nutrition has been related to several other conditions, ranging from pediatric cancers (Goh et al. 2007) to 
autism (Suren et al. 2013), I have limited this discussion to the realm of growth, obesity and metabolic dysregulation. Despite the wide acceptance that maternal nutrition plays a significant role in programming offspring health, a possible role for paternal nutrition has been mostly ignored. Given scale of the obesity epidemic facing countries like the US, all possible avenues need to be considered to combat this worrying trend which continues unabated; including the optimization of both maternal and paternal nutrition in order to prevent the transmission of detrimental metabolic phenotypes to offspring that may pre-dispose to obesity and insulin resistance (IR).

\section{Maternal}

\section{Human Studies}

Many of the human studies investigating the relationship between maternal B vitamin status and offspring growth and metabolism has been conducted in the India and The Netherlands.

Perhaps the first demonstration that maternal B vitamin status is related to risk factors for metabolic disease in offspring comes from the Pune Maternal Nutrition Study(Yajnik et al. 2008). Women residing in six rural villages near Pune were assessed for anthropometry, physical workload, dietary intake and various blood nutrients at 18 and 28 weeks of gestation. Their offspring were measured with 72 hours of birth and followed until 6 years of age. Low maternal folate and higher homocysteine concentrations were associated with smaller newborn weight while $B_{12}$ and methylmalonic acid (MMA) status were unrelated. Higher maternal RBC folate was associated with higher adiposity and higher insulin resistance (HOMA-IR), while lower maternal vitamin $B_{12}$ predicted higher IR in 6 year old children. Interestingly, offspring of mothers with high folate and low $B_{12}$ were the most insulin resistant.

A similar study was conducted at the Holdsworth Memorial Hospital in Myosore, Southern India(Krishnaveni et al. 2014). Maternal plasma vitamin $\mathrm{B}_{12}$, folate and homocysteine were measured at around 30 weeks of gestation in 654 women with a live birth. The majority of the children of these mothers were assessed at 5, 9.5 and 13.5 years of age for 
anthropometry and IR. After adjusting for maternal and offspring covariates including current BMI, maternal blood folate concentrations were positively associated, while $B_{12}$ concentrations were negatively associated with fasting glucose concentrations in their offspring measured at 5 years of age. Maternal folate, but not $B_{12}$ concentrations remained significantly related to fasting insulin and HOMA-IR in children at both 9.5 and 13.5 years of age.

Thus the findings from Myosore and Pune are in good agreement regarding higher maternal folate status and lower $\mathrm{B}_{12}$ being related to higher offspring IR in 5-6 year old children.Unlike the Pune study, where children of mother with high folate and low $B_{12}$ were the most insulin resistant, there did not appear to be an interaction between maternal folate and $B_{12}$ status in the Myosore study.One possible explanation for the discrepancy between Myosore and Pune studies regarding $B_{12}$ is differences may lie in the timing of maternal sampling. In the Pune study, analyses revealed that mid gestation (18 weeks) vitamin $B_{12}$, but late gestation ( 28 weeks) folate concentrations were the strongest predictors of IR (Yajnik et al. 2008). Because the Myosore study sampled mothers at around 30 weeks of gestation they may have missed the window for detecting the $B_{12}$ association. Another point that these studies raise is the possibility that the effect of maternal $\mathrm{B}_{12}$ on offspring is transient and may lessen over time.

Further evidence for a role of vitamin $B_{12}$ in predicting offspring insulin resistance comes from a trial of antenatal micronutrient supplements in Nepal. Pregnant mothers were cluster-randomized mice to receive either control or 400 $\mu \mathrm{g}$ folic acid with or without additional iron, zinc and other micronutrients (Christian et al. 2003; Stewart et al. 2011). The mean gestational age at participation was 10.2 weeks, with mothers being sampled again at 32.6 weeks. Their children were assessed at $6-8$ years of age.Unlike the Indian studies, maternal folate status - measured either early or late in pregnancy- was not related to HOMA-IR in children at 6-8 years of age. In contrast, mothers who had low $B_{12}$ status ( $<148 \mathrm{pmol} / \mathrm{L}$ ) had children with a HOMA-IR $27 \%$ higher than children of $B_{12}$ sufficient mothers. Importantly, and in agreement with the Pune study(Yajnik et al. 2008), while early $B_{12}$ status was related to offspring IR, late $B_{12}$ status was unrelated. In contrast, data from Mysore also showed that late gestation (30wk) $B_{12}$ impacted offspring IR, but only at 5 years of age and not at 9.5 pr 13.5 years of age (Krishnaveni et al. 2014) 
In addition to metabolic markers, low offspring birthweight has been studied in regard to maternal folate and $B_{12}$ status. Although sometimes used interchangeably, small for gestational age (SGA) specifically refers to those newborns below the $10^{\text {th }}$ percentile of body weight for a specific gestational age according to World Health Organization standards (WHO 1995). While SGA may simply occur because a child is constitutionally small, Intrauterine Growth Restriction (IUGR) refers to conditions where the maximal potential size of a fetus cannot be reached due to maternal nutritional deficiencies, poor weight gain during pregnancy,hypertension and preeclampsia among other conditions (Sharma et al. 2016). These outcomes are highly prevalent in India where one third of babies are of low birth weight $(<2.5 \mathrm{~kg})$. In contrast the incidence of low birth weight is around 6-7\% in North America and Europe (Wardlaw et al. 2004). Low birthweight outcomes are of interest because they may predispose to metabolic disorders such as obesity, type 2 diabetes, and metabolic syndrome in later life (Yajnik et al. 1995; Hales and Barker 2001; Whincup et al. 2008).

In Bangalore, Southern India, a prospective study was conducted at St. Johns Medical Center Hospital to investigate the potential link between maternal folate and $B_{12}$ status and IUGR (which they also defined as birth weight below the $10^{\text {th }}$ percentile for gestational age). 478 women were recruited and assessed at the $1^{\text {st }}, 2^{\text {nd }}$ and $3^{\text {rd }}$ trimester. Almost $30 \%$ of newborns in this cohort were affected by IUGR. Women in the lowest two tertiles for $B_{12}$, especially during the $2^{\text {nd }}$ trimester, had a significantly higher risk of having a child with IUGR (Adjusted Odds ratio $2.8-9.28$ ). Red blood cell (RBC) folate was not associated with IUGR at any time point during pregnancy, however it was not reported whether an interaction between folate and $B_{12}$ was studied (Muthayya et al. 2006).

In a second but expanded study of the Bangalore cohort, Dwarkanathet a/tested whether unbalanced intakes of folate and $B_{12}$ during pregnancy can affect the incidence of births affected by SGA(Dwarkanath et al. 2013).Amongst the 1838 live births SGA was significantly and negatively associated with plasma folate and $B_{12}$ in $1^{\text {st }}$ trimester but not at later time points. Interestingly, at the second trimester a $\mathrm{B}_{12}$-folate interaction emerged; subjects with high folic acid intake $\left(>1000 \mu \mathrm{g} / \mathrm{d}\right.$ ) but in the lower two tertiles of $\mathrm{B}_{12}$ had a $\sim 2.7$ fold higher risk of having children affected by SGA. Similarly in the Pune study, low maternal folate was also associated with low birthweight in offspring (Yajnik et al. 2008). 
Also in the southern hemisphere, researchers in South Australia conducted a retrospective study to assess the relationship between $1^{\text {st }}$ trimester RBC folate concentrations and risk for SGA. The found that the RBC folate of mothers with SGA children was $22 \%$ lower than that of mothers with normal weight children. Moreover, folate insufficiency (221 $-400 \mathrm{nmol} / \mathrm{L}$ ) and deficiency (<200 $\mathrm{nmol} / \mathrm{L}$ ) were associated with stepwise increase in odds ratio of having SGA child. Unfortunately plasma $B_{12}$ were not measuredin this study (Furness et al. 2012).

Several studies investigating the role of maternal nutrition in the programming of offspring growth and metabolism have also been conducted in Europe. In the Amsterdam Born Children Study (ABCS) maternal serum vitamin $B_{12}$ and folate were measured in the $1^{\text {st }}$ trimester and related to various outcomes(Krikke et al. 2016). Mothers in the lowest quartile for serum folate had children with a higher BMland systolic blood pressure at 5-6 years but birthweight was unchanged. In addition, children of mothers in the lowest quartile of serum $B_{12}$ displayed a higher heart rate at 5-6 years. Unlike the Indian cohorts no effect of maternal folate or $\mathrm{B}_{12}$ was seen on offspring blood glucose measures. One factor that may have made it more challenging to detect such effects in this Dutch population is the relatively high degree of heterogeneity of the mothers sampled and their apparent large differences in one-carbon nutrient status: for example women of Ghanaian decent had the highest $(600 \mathrm{pg} / \mathrm{ml})$ while Turkish women had the lowest $(204 \mathrm{pg} / \mathrm{ml})$ median serum $\mathrm{B}_{12}$ concentrations. Interestingly, despite having the highest serum $\mathrm{B}_{12}$ concentrations, Ghanaian women had the lowest median folate concentrations $(11.8 \mathrm{nmol} / \mathrm{L})$ while Dutch women had the highest $(26 \mathrm{nmol} / \mathrm{L})$.

In the Generation R study, also in the Netherlands, folic acid supplementation was evaluated as a potential modifier of birth weight and incidence of pre-term birth. Starting supplementation preconception was associated with a significant elevation of birthweight and placental weight compared to no supplementation. Moreover, starting supplementation before and after pregnancy confirmation were both associated with a reduced risk for low birth weight $(<2.5 \mathrm{~kg})$ and SGA (Timmermans et al. 2009). In another analysis of this cohort maternal plasma folate was measured at 13 weeks of gestation and offspring head circumference measured repeatedly during fetal life and up to 6 years in 5866 children (Steenweg-de Graaff et al. 2015). Increasing maternal folate associate with modestly faster prenatal head size and growth but postnatal growth was unaffected. 
In the Rotterdam 'PREDICT' study, van Uitertet al studied the relationship between maternal folate status and embryonic growth(van Uitert et al. 2014). They report that women in the $3^{\text {rd }}$ tertile of RBC folate had embryos significantly larger, as determined by crown-rump length, than offspring of mothers in the lower two quartiles of folate. Interestingly, mothers with the highest folate ( $4^{\text {th }}$ quartile) had offspring that were $24 \%$ smaller at 6 weeks and $7.6 \%$ smaller at 12 weeks of gestation. These data suggest that there may be an optimal folate concentration to support growth, beyond which suppression of growth may occur. It is unclear however, whether such effects may persist beyondbirth and the reduced difference at 12 compared to 6 weeks of gestation may suggest that the effect diminishes over time.

In the UK, Lewis et al investigated the relationship between maternal folate intake and offspring body composition at 9 years of age using the Avon Longitudinal Study of Parents And Children (ALSPAC)(Lewis et al. 2009). Use of folic acid supplements was recorded at 18 and 32 weeks gestation and dietary folate intake was estimated from self-administered FFQs at 32 weeks gestation. There was no difference in the body composition of children of mother who used compared who refrained from folic acid supplements at both time points. There was however a weak $(P=0.04)$ positive association between folate intake and lean body mass that the authors predicted to be artefactual. Unfortunately blood folate and $\mathrm{B}_{12}$ concentrations were not available for this study as these measuresare a far more precise measure of folate status and, as such, may have provided an increased power to detect any effects on offspring growth. Also other studies suggest that effects of maternal one-carbon status may be evident earlier in childhood but not later.

Perhaps the most consistent finding from these various birth cohorts regards low birthweight; at least 4 studies over 3 continents suggest that low maternal blood folate concentrations are associated with lower offspring body weight and higher risk for having a SGA child (Yajnik et al. 2008; Timmermans et al. 2009; Furness et al. 2012; Dwarkanath et al. 2013). Thus, in addition to low maternal folate status being associated with an increased risk for neural tube defects in children (Lumley et al. 2001), it appears that more subtle metabolic changes are also invoked that culminate in reduced 
fetal growth. As discussed above, low birthweight is a public health concern because it may predispose to metabolic disease and obesity later in life (Yajnik et al. 1995; Hales and Barker 2001; Whincup et al. 2008).

Evidence is accumulating, however, that more folate is not better and there has been concern for some time that excessive folate intake may induce deleterious effects (Selhub and Rosenberg 2016).Although the birth cohort data for high folate is less consistent than for low intakes, there is some indication that high folate may also induce metabolic and growth disturbances. For example in the Rotterdam PREDICT study women in the highest quartile of folate carried fetuses of reduced size compared to those in the third quartile (van Uitert et al. 2014). A particularly intriguing aspect of the high folate story is the apparent interaction with $\mathrm{B}_{12}$; for example in the Pune study high folate was associated with IR, but it was the mothers with both high folate and low $B_{12}$ that had children with the highest IR (Yajnik et al. 2008). Similarly, in the Bangalore cohort mothers with the highest folate and lowest $\mathrm{B}_{12}$ concentrations had the highest risk of having a SGA-affected child (Dwarkanath et al. 2013).Although not measured in all cohorts, the importance of adequate maternal $B_{12}$ status is becoming evident; low maternal $B_{12}$ was associated with increased insulin resistance in offspring in the Pune (Yajnik et al. 2008), Myosore(Krishnaveni et al. 2014) and Nepal (Stewart et al. 2011) studies and with SGA in the Bangalore cohort (Dwarkanath et al. 2013). Moving forward it is imperative that these two critical interrelated onecarbon nutrients be considered when studying maternal programming of offspring health.

\section{Rodent Studies}

To date, over a dozen studies have been conducted to investigate the effect of altered maternal one-carbon nutrient intake on offspring growth and metabolism using rodents. In rodents the basal requirement of folate is $2 \mathrm{mg}$ folic acid /kg diet (Reeves et al. 1993)and at the simplest level these studies can be broken down into those intervening with diets containing more (supplemental) or less (deficient) than this basal amount. Further complexity is added to this body of literature with the modulation of multiple one-carbon nutrients and by also altering offspring diet. Moreover, altering the duration and window of exposure to a specific diet is also a key variable to be considered. Given these factors, there 
are hundreds of different combinations of intervention designs possible and distilling central themes from this body literature is challenging.

\section{Deficiency Studies}

As described above, the human literature on maternal folate status has quite consistently demonstrated that low maternal folate status is associated with low birthweight but not IR. Regarding the latter phenotype, it appears that plasma $\mathrm{B}_{12}$ status may actually a stronger determinant than folate.

To test the impact of maternal folate insufficiency Maloney et al removed folate from the diet of female rats beginning two weeks before mating and continuing through pregnancy and weaning. They retained offspring until 24 weeks of age and noted that female offspring of folate deficient mothers were $6 \%$ lighter than those of control mothers. Interestingly, there was no similar effect observed in males and glucose and insulin concentrations were unaltered in both sexes of offspring (Maloney et al. 2009).

Similarly McKay et al fed a low folic acid $(0.4 \mathrm{mg} / \mathrm{kg})$ diet to female C57BI/6 mice for 4 weeks before mating and through pregnancy and weaning. In addition,offspring were randomized to be fed low or high fat diets and retained until 26 weeks of age. Maternal folate depletion had no effect on offspring body weight or adiposity, although blood glucose concentrations were modestly lower. This latter observation is agrees with the Myosore study in which maternal folate status was found to be positively associated with offspring blood glucose concentrations (Krishnaveni et al. 2014).Interestingly, an interaction between maternal and offspring diets emerged; offspring fed a high fat diet had $30 \%$ elevated plasma triglycerides, but only when born to low folate dams (McKay et al. 2014). Such an interaction has not yet been observed in human studies.

In a second study by Maloney et al, female rats were fed a 'methyl-deficient' diet devoid of folic acid and low in methionine and choline three weeks before mating and though gestation day 5 . Amongst female offspring there was no effect of maternal diet on HOMA-IR or response to oGTT at 6 months of age. In contrast, male offspring of deficient 
dams had elevated HOMA-IR and peak glucose after OGTT; an effect that persisted until 12 months of age. (Maloney et al. 2011).

As described above, the human data suggest that maternal $B_{12}$ status is also associated with birthweight and IR in offspring. To test this in rodents Kumar et al placed female Wistar rats on control, low folate, low $B_{12}$ or low folate and $\mathrm{B}_{12}$ diets for 3 months before mating to control fed males. They report that, despite being born lighter, pups born to $B_{12}$ deficient dams surpassed the weight of control pups by weaning and were $50 \%$ heavier at 12 months of age. Interestingly, folate deficient pups had a normal birthweight but also quickly became heavier. Perhaps even more intriguing, at 12 months of age the percentage body fat was elevated by $35 \%$ in pups of $B_{12}$ deficient dams but by $300 \%$ in offspring of folate deficient dams - despite being a similar body weight(Kumar et al. 2013). Following up on this study, the investigators fed Wistar rats on replete or mildly $B_{12}$ deficient diets for 3 months before mating with controlfed males. Vitamin $B_{12}$ status was then restored in mothers or offspring by switching groups to control diet at conception, birth and weaning or not all. Compared to offspring of control-fed dams, those of deficient dams weaned onto a control diet were significantly lighter at birth but quickly became heavier with reduced lean mass and fat free mass through 9 months of age. Moreover, offspring of deficient mothers displayed elevated fasting insulin, HOMA-IR and blood glucose after oGTT(Kumar et al. 2014). Data from these two studies are in good agreement with those of the Bangalore study showing that low maternal $B_{12}$ was associated with an increased risk for low birth weight (Muthayya et al. 2006) and also with the Pune(Yajnik et al. 2008), Myosore(Krishnaveni et al. 2014), Nepal (Stewart et al. 2011)studies which showed that low maternal $B_{12}$ is associated with elevated HOMA-IR in offspring.

\section{Supplementation Studies}

With the usage of periconceptional folic acid supplements commonplaceand the implementation of folate fortification in several countries - both to combat neural tube defects - concern has gradually shifted away from the deleterious effects of low maternal folate status on offspring health to studying the possible adverse effects of supplemental or very high folate levelson the long term health and physiology of offspring.Data from the human birth cohorts in Pune (Yajnik 
et al. 2008)and Myosore(Krishnaveni et al. 2014) indicate that there may indeed be positive association between high maternal folate status and offspring adiposity and IR.

Huotet al fed female rats diets containing 2 or $5 \mathrm{mg} / \mathrm{kg}$ FA for 3 weeks before mating and weaned offspring from each maternal group onto 2 or $5 \mathrm{mg} / \mathrm{kg}$ folic acid. Amongst control-fed male offspring, those born to supplemented dams had significantly elevated body weight and IR compared to those of control dams. In contrast, when the male offspring were weaned onto the supplemented diet the effect of maternal supplementationon weight and IR was ablated. Again, sex differences in the response to maternal folate were noted; where male offspring of supplemented damswere heavier, female pups were lighter than control pups. Moreover, where offspring supplementation negated the increased weight and IR phenotype induced by maternal supplementation in male pups, in female pups the IR was actually precipitated by supplementation(Huot et al. 2016).

Although starting supplementation later at conception, Chmurzynskaet al also compared offspring of female rats fed 2 and $5 \mathrm{mg} / \mathrm{kg}$ folic acid. Subsequently, offspring were challenged with a high fat diet from 4 - 10 weeks of age or from 10 - 16 weeks of age.For the groups starting at 4 weeks, neither pre-natal nor post-natal diet affected body weight. In contrast, for the later groups maternal folate supplementation was associated with a $10 \%$ reduction in body weight (Chmurzynska et al. 2012).

Burdgeet al fed female rats diets containing 1 or $5 \mathrm{mg} / \mathrm{kg}$ folic acid starting at conception and continuing through birth and offspring were randomized to be fed low or high fat diets. They noted that offspring did not differ in weight due to maternal folic acid intake. Blood triglyceride concentrations were higher in offspring of high folate dams irrespective of their own diet but blood glucose was only elevated when high folate offspring consumed a high fat diet(Burdge et al. 2008). While choosing a non-standard folate concentration as the control makes direct comparisons to other studies difficult, the data does suggest that some offspring phenotypes may be latent and can be uncovered by an obesogenic offspring diet. 
Similarly, Huang et al fed female mice diets containing 2,5 or $40 \mathrm{mg} / \mathrm{kg}$ FA and of age male offspring were placed on a high fat diet for from 7-15 weeks of age. Offspring of the highest folic acid group displayed the highest body weight, largest amount of intra-abdominal and subcutaneous fat and highest HOMA-IR. Before the high fat challenge such differences were absent - suggesting that either time, an obesogenic diet or both are required to uncover this phenotype in mice (Huang et al. 2014).

Keating et al fed female rats 0,2 or $40 \mathrm{mg} / \mathrm{kg}$ FA starting at mating and lasting through pregnancy and lactation.They noted that supplementation was associated with heavier birthweight and in both male and female offspring but the differences became more apparent over 12 months in females and disappeared in males. Excess weight gain in females was associated with disrupted food intake characterized by several spikes in daily intake. Moreover at 3 months of age high folic acid offspring had elevated fasting blood glucose, while at 10 months post GTT glucose was elevated - in agreement with Pune (Yajnik et al. 2008) and Myosore(Krishnaveni et al. 2014). Finally, female but not male offspring of supplemented dams increased fat mass in response to fructose feeding while offspring of control dams did not (Keating et al. 2015). These data again highlight sex specificity in response and also that some phenotypes may be uncovered by addition challenge to the offspring.

Perhaps one of the most interesting findings from the human literature is the apparent interaction of maternal high folate and low $B_{12}$ concentrations - a combination that was associated with increased IR (Yajnik et al. 2008; Krishnaveni et al. 2014) and SGA (Dwarkanath et al. 2013). To the best of my knowledge only one study has attempted to evaluate this scenario in rodents; female mice were fed one of three diets containing control folic acid and $B_{12}(50 \mu \mathrm{g} / \mathrm{kg} \mathrm{diet})$, high folic acid $\left(10 \mathrm{mg} / \mathrm{kg}\right.$ ) with control $B_{12}$ or high folic acid without $B_{12}$ starting 6 weeks before mating and lasting through weaning.Male offspring were weaned onto a control or western diet. Amongst control-fed offspring, thoseof high folic acid $+B_{12}$ dams displayed the greatest weight gain while offspring of high folic acid without $B_{12}$ displayed reduced insulin concentrations. There was no effect of maternal diet on offspring glucose tolerance or baseline glucose concentrations in offspring fed control or western diets (Aleliunas et al. 2016). Thus, despite being designed to mimic the conditions of the Indian birth cohorts, this study failed to corroborate the findings in regard to birthweight and 
offspring IR. Several reasons for this discrepancy are possible, including; insufficient duration of maternal intervention, inter-species differences and the possibility that offspring may also need to have inadequate $B_{12}$ status - a highly prevalent situation in India (Refsum et al. 2001)- for the IR phenotype to emerge. Moreover, although maternal $B_{12}$ concentrations were confirmed to be reduced in mothers fed the $B_{12}$ deficient diets, blood folate concentrations of dams were not measured and we cannot, therefore, assess the degree to which folate concentrations were elevated and compare these differences to the ranges seen in the human studies. The study did however observe an increased weight gain in offspring of folic acid supplemented dams. A similar response to high FA was also seen in male offspring of femalerats fed a slightly lower supplemental dose $(5 \mathrm{mg} / \mathrm{kg})$, but in that study maternal folic acid supplementation was also associated with elevated IR (Huot et al. 2016)- the latter finding agreeing with those on IR in Pune (Yajnik et al. 2008) and Myosore(Krishnaveni et al. 2014). This discrepancy highlights the interspecies differences in response observed even with rodents, and suggest that, even though C57BL6J mice are considered a good model for human metabolic disease (Collins et al. 2004), they may be less accurate for modelling parental programming.

In addition to studying the modulation of maternal folic acid $B_{12}$ intakes, researchers have evaluated the effect of multiple methyl vitamin supplementation on offspring health and, given that expectant mothers often take multivitamins rather than folic acid alone (Shand et al. 2016), these studies may have even greater public health relevance than those looking at single nutrients. In one such study Cho et al fed female rats diets containing $10 x$ the basal requirement for folic acid, vitamin $B_{12}$ and vitamin $B_{6}$ from the $3^{\text {rd }}$ day of pregnancy until term and then weaned male offspring onto a high fat diet. They noted that offspring of high methyl vitamin dams ate more,were significantly heavier and had a higher glucose response than offspring of control dams. When they repeated the study with high maternal vitamins but basal folic acid that the found that growth and glucose differentials were absent - indicating an essential role for folic acid (Cho et al. 2015).That maternal folic acid supplementation induced increased weight gain and IR in offspring is in agreement with other rat (Keating et al. 2015)and human studies (Yajnik et al. 2008)(Krishnaveni et al. 2014). 
Giudicelliet al tested the additional methyl donors methionine, choline and betaine and found supplementing female rats with the combination resulted in female offspring with decreased birth weight and decreased plasma triglycerides and increased fasting glucose at 3 weeks of age -changes absent in male offspring(Giudicelli et al. 2013). Similarly Huotet al observed a weight reduction (Huot et al. 2016) while Keating et al observed higher plasma glucose in female offspring (Keating et al. 2015) of supplemented dams.

Despite the use of single or multiple vitamins at varied doses and starting and ending at different times, as well as the weaning of different sexed offspring onto different diets a few common themes have begun to emerge from this body of literature. Firstly, five studies indicate that high maternal folic acid intake is associated with elevated plasma glucose concentrationsor IR in offspring although this not clear cut; some studies show this only in male offspring (Huang et al. 2014; Cho et al. 2015; Huot et al. 2016), others only in female offspring (Giudicelli et al. 2013)and only one demonstrating this in both sexes (Keating et al. 2015). Thus, the second point is that the response to high maternal folic acid intake appears to be sex-specific. For example, in one study high glucose was seen only in male offspringweaned onto a control diet but not when weaned onto a high folic acid diet. The opposite was seen in female pups; those weaned onto control diet had normal glucose but those weaned onto high folic acid displayed IR (Huot et al. 2016).

This brings up the final point; there appears to be an interaction between maternal and offspring diet - or at least a some additional challenge may be required to uncover a latent phenotype in offspring. For example, offspring blood glucose was elevated only when fed a high fat diet (Burdge et al. 2008). Moreover, in two of the studies demonstrating that maternal high folic acid induced high glucose in male offspring they were all weaned onto a high fat diet (Huang et al. 2014; Cho et al. 2015).

\section{Paternal}

Unlike maternal one-carbon status, very little attention has been paid to how paternal one-carbon status may affect offspring health and metabolism. Nevertheless, there is a small but growing body of literature that demonstrates proof- 
of-concept that paternal one-carbon nutrient status may also be a determinant of offspring health and disease. For example, paternal folate deficiency in rats reduced body weight, length, hepatic folate and brain lgf2 expression in fetal offspring (Kim et al. 2013).Our own studies, although initially designed to study tumorigenesis, show that paternal B vitamin intake can also induce changes in body weight and metabolism in offspring. Female offspring of B vitamin (vitamins $B_{2,6,12}$ and folic acid) supplemented and deficient mothers were both significantly lighter throughout their life(6 mo) than pups of control fed dams. Hepatic transcriptome analyses suggested a disruption of lipid metabolism and subsequent analyses revealed that despite being lighter, female offspring of supplemented sires had an approximately three-fold elevation in hepatic triglyceride content. Sex-specific re-analysis of our RNA-seq data revealed a significant upregulation of three genes (Acaca, Elovl6, Gpam), the products of which are sequentially involved in de novo triglyceride synthesis in the liver(Sabet et al. 2016). Thus these data also suggest that paternal on-carbon status may also be a determinant of offspring metabolism, and raise the intriguing possibility of an interaction of maternal and paternal diet in programming offspring health. 


\section{Conclusions}

There is a substantial body of literature which pertains to examining to the effect of differing maternal folate status on offspring health and metabolism in both human cohorts and rodent models. Careful review of this literature, although highly heterogeneous, has uncovered several themes.

Firstly,taken together, data from two Indian birth cohorts (Yajnik et al. 2008; Krishnaveni et al. 2014) as well as five rodent studies(Giudicelli et al. 2013; Huang et al. 2014; Cho et al. 2015; Keating et al. 2015; Huot et al. 2016)strongly suggest that high maternal folic acid intake is associated with elevated blood glucose or IR in offspring. The strength of the association is somewhat tempered by caveats in the rodent data (sex specificity and interactions with offspring diet), nevertheless taken together the body of literature remains credible.As a result of folate fortification and the widespread use of vitamin supplements ( $35 \%$ of US adults use vitamins containing folic acid), a relatively large proportion of the US population consumes "high" amounts of folate. Analysis of the NHANES (2003 - 2006) data set showed that $25 \%$ of women aged $19-50$ years consume around twice the RDA for women (400 $\mu \mathrm{g} / \mathrm{d})$ or $1.33 \mathrm{x}$ the RDA of pregnant women $(600 \mu \mathrm{g} / \mathrm{d})$. Moreover $5 \%$ of women this age consume around $4 x$ the RDA of dietary folate equivalents (Bailey et al. 2010).

Secondly, findings from several studies establish the critical importance of maternal $B_{12}$ sufficiency for offspring health. Specifically, studies in both humans (Yajnik et al. 2008; Stewart et al. 2011; Krishnaveni et al. 2014) and rats (Kumar et al. 2014 ) indicate that maternal $\mathbf{B}_{12}$ insufficiency is associated with elevated IR in offspring. Moreover, data from human (Muthayya et al. 2006; Dwarkanath et al. 2013) and rat studies (Kumar et al. 2013; Kumar et al. 2014) consistently show that low maternal $\mathbf{B}_{\mathbf{1 2}}$ is associated with reduced birthweight. These observations fit well together because low birthweight is a known risk factor for metabolic disease later in life (Yajnik et al. 1995; Hales and Barker 2001; Whincup et al. 2008). These findings are of importance because $B_{12}$ deficiency remains prevalent in many populations; for example low plasma $\mathrm{B}_{12}(<150 \mathrm{pmol} / \mathrm{l})$ was observed in $60 \%$ of pregnant women in the Pune study (Refsum et al. 2001) and 17 35\% of pregnant women in a Canadian cohort (Visentin et al. 2016). 
For more than two decades the US Centers for Disease Control (and Prevention) have recommended that women anticipating pregnancyshould consume $400 \mu \mathrm{g}$ folic acid/day to minimize the risk of their pregnancy being complicated by a neural tube defect (NTD) (CDC 1992). Moreover, the US Food and Drug Administration has required the addition of folic acid to enriched grain products since 1996 (FDA 1996) - a public health intervention that is credited with eliciting an approximately $35 \%$ in the incidence of NTDs in the US (Williams et al. 2015). Several leaders in the field on one-carbon metabolism have called for these programs to be expanded to include vitamin $B_{12}$ in order to combat poor $B_{12}$ status (which remains pervasive) and anemia, as well as memory and cognitive impairment (Refsum and Smith 2008; Allen et al. 2010; Selhub and Paul 2011). The increasing acceptance that maternal nutrition is a determinant of offspring obesity and IR(Nicholas et al. 2016), together with data from humans (Yajnik et al. 2008; Stewart et al. 2011; Krishnaveni et al. 2014) and rodents (Kumar et al. 2014) indicating that low maternal $B_{12}$ status is associated with elevated offspring IR, adds a new dimension to this call for $\mathrm{B}_{12}$ fortification. Although cut-offs for defining insulin resistance vary, the condition clearly is highly prevalent and remains a major public health issue in the US. In NHANES (1999-2002)the prevalence of IR amongst US adults was estimated to be $32 \%$ when applying a cutoff of HOMA-IR $>2.35$ (Ioannou et al. 2007). Because efforts to tackle obesity and IR have largely been ineffective (Mitchell et al. 2011), new public health initiatives that look beyond an individual's own diet, activity and genetic make-up may be warranted. Specifically, a novel avenue for addressing the obesity epidemic may be to optimize maternal pre-conception nutrient status to reduce the risk of instilling detrimental metabolic programs in offspring. Given that $B_{12}$ has no demonstrated toxicity (and has no specified tolerable upper limit), insufficiencies remain pervasive (Refsum et al. 2001; Visentin et al. 2016) and human and mouse data both link low maternal $B_{12}$ to elevated IR in offspring, fortification - or at least formal recommendations to supplement with this vitamin -should be seriously considered. An added benefit of $B_{12}$ fortification may be to reduce the risk of cognitive decline and dementia amongst the elderly; poor $\mathrm{B}_{12}$ status (assessed by methylmalonic acid and holotranscobalamin) is associated with an elevated risk of these condition in the elderly (O'Leary et al. 2012).

Thirdly, the human data is relatively consistent in reporting that low maternal folate status is associated with low birthweight(Yajnik et al. 2008; Timmermans et al. 2009; Furness et al. 2012; Dwarkanath et al. 2013). Given the consistency of this fining, it is quite surprising then that a similar theme has not emerged from the rodent data. Although 
one study has shown low maternal folate to reduce the weaning weight of pups (McKay et al. 2014), others have shown no effect on birthweight (Kumar et al. 2013) or even a heavier fetus weight in rats (Maloney et al. 2009). The reasons for this discordance between the human and rodent data and even between the rodent studies are unclear but may include the degree and duration of depletion as well as interspecies differences in energy and folate metabolism. Clearly further work is required to better model the human situation in rodents and subsequently elucidate mechanisms.Although reduced since the introduction of fortification, $15-17 \%$ of women aged $19-50$ still consume less than the estimated average requirement (320 $\mathrm{\mu g} / \mathrm{d}$ DFE) of folate (Bailey et al. 2010).

\section{Recommendations for Future Research}

As discussed above, three themes have emerged from the literature on maternal one-carbon status and offspring growth and metabolism: 1) high maternal folate status is associated with elevated IR in offspring, 2) low maternal $B_{12}$ status is associated with elevated IR and reduced birthweight and 3) low maternal folate status is associated with reduced birthweight. One striking aspect of the animal literature is the high degree of heterogeneity of approach, which when one considers the number of possible variables in designing an intervention, is not surprising. Specifically,studies differ in the length and timing of supplementation, magnitude and composition of vitamin supplementation and depletion as well as the micro- and macro-nutrient composition of offspring diet. Despite this heterogeneity the rodent data is in good agreement with human finings with regard to maternal high folate and low $B_{12}$ status both being associated with elevated IR in offspring.

Moving forward, it is suggested that the design of human studies should be informed by animal findings and vice versa. Key elements in the rodent data, that remain understudied in humans, are the differential effect of maternal diet on metabolism in male and female offspring as well as potential interactions with offspring diet. 
One intriguing observation of human studies is the finding that children of mothers with high folate and low $B_{12}$ had the highest IR (Yajnik et al. 2008; Krishnaveni et al. 2014). Only one group has attempted to model this scenario in rodents but were unable to recreate the elevated IR in male offspring that was seen in children (data from female pups were not reported)(Aleliunas et al. 2016). This interaction of high folate and low $B_{12}$ is a particularly fascinating topic in the onecarbon field. Observations that individuals with high folate and low $B_{12}$ have the highest blood homocysteine and methylmalonic acid concentrations, functional markers of poor folate and $B_{12}$ status (Miller et al. 2009), as well as elevated prevalence of anemia and accelerated cognitive decline (Morris et al. 2012) have given rise to the hypothesis that high folic acid intake exacerbates the detrimental effects of $B_{12}$ insufficiency.Given that high folate intakes are common and $B_{12}$ insufficiency remains pervasive there appears to a real public health issue that remains understudied and as a result poorly understood. It is my suggestion that future rodent studies be carefullydesigned in light of observations from human studies to thoroughly test this hypothesis in the maternal and paternal setting.

Another aspect of this field that, in my opinion, is worthy of further attention is the potential role for paternal onecarbon status in programming offspring metabolism. Given the constant production of sperm through adult life, the integrity of the genetic and epigenetic information carried within is constantly at the mercy of an adequate nutrient supply. Indeed, we (Sabet et al. 2016) and others (Lambrot et al. 2013) have recently demonstrated that altering paternal B vitamin intake can cause widespread changes in the sperm methylome. It remains to be seen which, if any, of these methylation changes escape the widespread demethylation that occurs during embryogenesis - a task that will require single base resolution comparisons of sperm and offspring methylomes. Alternatively, other epigenetic moieties such as miRNA may play a role in the transmission of phenotypes to offspring and also need to be considered.

Finally, a host of offspring phenotypes have been associated with maternal and paternal one-carbon nutrient intake using both human cohorts and rodent interventions. One thing that is apparent is that we have poor mechanistic understanding of the changes that underpin the observed phenotypic changes, and especially in the paternal setting, how the information is transmitted from father to child. Although changes have been observed in the expression and methylation of Igf2 and Ppar $\alpha$ that associate with phenotypes, causality is lacking. Moreover, it is likely that mechanisms 
involved are far more complicated that changes in these two likely candidates. One factor that will continue to hamper studies in this field, as well as the broader field of epigenetics, is our inability to engineer specific methylation changes into cells. Unlike genetic engineering where it is possible to knock out, knock down or mutate genes, we are unable to do little more than elicit genomic hypomethylation through the use of 5-aza-cytidine. This means that any changes we seen in the methylation of candidate genes, no matter how plausible, are likely remain mere associations until we figure out how to alter the methylation status of specific $\mathrm{CpG}$ residues to test their mechanistic involvement in any phenotype. 


\section{References}

Aleliunas, R. E., A. M. Aljaadi, et al. (2016). "Folic Acid Supplementation of Female Mice, with or without Vitamin B-12, before and during Pregnancy and Lactation Programs Adiposity and Vascular Health in Adult Male Offspring." J Nutr.

Allen, L. H., I. H. Rosenberg, et al. (2010). "Considering the case for vitamin B12 fortification of flour." Food Nutr Bull31(1 Suppl): S36-46.

Bailey, R. L., K. W. Dodd, et al. (2010). "Total folate and folic acid intake from foods and dietary supplements in the United States: 2003-2006." Am J Clin Nutr91(1): 231-237.

Barker, D. J. and C. Osmond (1986). "Infant mortality, childhood nutrition, and ischaemic heart disease in England and Wales." Lancet1(8489): 1077-1081.

Barker, D. J., P. D. Winter, et al. (1989). "Weight in infancy and death from ischaemic heart disease." Lancet2(8663): 577580.

Burdge, G. C., K. A. Lillycrop, et al. (2008). "The nature of the growth pattern and of the metabolic response to fasting in the rat are dependent upon the dietary protein and folic acid intakes of their pregnant dams and post-weaning fat consumption." Br J Nutr99(3): 540-549.

CDC (1992). Centers for Disease Control. Recommendations for the use of folic acid to reduce the number of cases of spina bifida and other neural tube defects. Morbidity and Mortality Weekly Report; Recommednations and Reports. 41: 1.

Chmurzynska, A., M. Stachowiak, et al. (2012). "Protein and folic acid content in the maternal diet determine lipid metabolism and response to high-fat feeding in rat progeny in an age-dependent manner." Genes Nutr7(2): 223234.

Cho, C. E., E. Pannia, et al. (2015). "Methyl vitamins contribute to obesogenic effects of a high multivitamin gestational diet and epigenetic alterations in hypothalamic feeding pathways in Wistar rat offspring." Mol Nutr Food Res59(3): 476-489.

Christian, P., S. K. Khatry, et al. (2003). "Effects of alternative maternal micronutrient supplements on low birth weight in rural Nepal: double blind randomised community trial." BMJ326(7389): 571. 
Collins, S., T. L. Martin, et al. (2004). "Genetic vulnerability to diet-induced obesity in the C57BL/6J mouse: physiological and molecular characteristics." Physiol Behav81(2): 243-248.

Cooney, C. A., A. A. Dave, et al. (2002). "Maternal methyl supplements in mice affect epigenetic variation and DNA methylation of offspring." J Nutr132(8 Suppl): 2393S-2400S.

Dwarkanath, P., J. R. Barzilay, et al. (2013). "High folate and low vitamin B-12 intakes during pregnancy are associated with small-for-gestational age infants in South Indian women: a prospective observational cohort study." Am J Clin Nutr98(6): 1450-1458.

FDA (1996). Food Standards: Amendment of Standards of Identity For Enriched Grain Products to Require Addition of Folic Acid, Food and Drug Administration. 66: 8781-8797.

Furness, D. L., N. Yasin, et al. (2012). "Maternal red blood cell folate concentration at 10-12 weeks gestation and pregnancy outcome." J Matern Fetal Neonatal Med25(8): 1423-1427.

Giudicelli, F., A. L. Brabant, et al. (2013). "Excess of methyl donor in the perinatal period reduces postnatal leptin secretion in rat and interacts with the effect of protein content in diet." PLoS One8(7): e68268.

Goh, Y. I., E. Bollano, et al. (2007). "Prenatal multivitamin supplementation and rates of pediatric cancers: a metaanalysis." Clin Pharmacol Ther81(5): 685-691.

Hales, C. N. and D. J. Barker (1992). "Type 2 (non-insulin-dependent) diabetes mellitus: the thrifty phenotype hypothesis." Diabetologia35(7): 595-601.

Hales, C. N. and D. J. Barker (2001). "The thrifty phenotype hypothesis." Br Med Bull60: 5-20.

Huang, Y., Y. He, et al. (2014). "Maternal high folic acid supplement promotes glucose intolerance and insulin resistance in male mouse offspring fed a high-fat diet." Int J Mol Sci15(4): 6298-6313.

Huot, P. S., A. Ly, et al. (2016). "Maternal and postweaning folic acid supplementation interact to influence body weight, insulin resistance, and food intake regulatory gene expression in rat offspring in a sex-specific manner." Appl Physiol Nutr Metab41(4): 411-420.

loannou, G. N., C. L. Bryson, et al. (2007). "Prevalence and trends of insulin resistance, impaired fasting glucose, and diabetes." J Diabetes Complications21(6): 363-370. 
Keating, E., A. Correia-Branco, et al. (2015). "Excess perigestational folic acid exposure induces metabolic dysfunction in post-natal life." J Endocrinol224(3): 245-259.

Kim, H. W., K. N. Kim, et al. (2013). "Effects of paternal folate deficiency on the expression of insulin-like growth factor-2 and global DNA methylation in the fetal brain." Mol Nutr Food Res57(4): 671-676.

Krikke, G. G., I. J. Grooten, et al. (2016). "Vitamin B12 and folate status in early pregnancy and cardiometabolic risk factors in the offspring at age 5-6 years: findings from the ABCD multi-ethnic birth cohort." BJOG123(3): 384392.

Krishnaveni, G. V., S. R. Veena, et al. (2014). "Association between maternal folate concentrations during pregnancy and insulin resistance in Indian children." Diabetologia57(1): 110-121.

Kumar, K. A., A. Lalitha, et al. (2013). "Maternal dietary folate and/or vitamin B12 restrictions alter body composition (adiposity) and lipid metabolism in Wistar rat offspring." J Nutr Biochem24(1): 25-31.

Kumar, K. A., A. Lalitha, et al. (2014). "Chronic maternal vitamin B12 restriction induced changes in body composition \& glucose metabolism in the Wistar rat offspring are partly correctable by rehabilitation." PLoS One9(11): e112991.

Lambrot, R., C. Xu, et al. (2013). "Low paternal dietary folate alters the mouse sperm epigenome and is associated with negative pregnancy outcomes." Nat Commun4: 2889.

Lewis, S. J., S. Leary, et al. (2009). "Body composition at age 9 years, maternal folate intake during pregnancy and methyltetrahydrofolate reductase (MTHFR) C677T genotype." Br J Nutr102(4): 493-496.

Lumley, J., L. Watson, et al. (2001). "Periconceptional supplementation with folate and/or multivitamins for preventing neural tube defects." Cochrane Database Syst Rev(3): CD001056.

Maloney, C. A., S. M. Hay, et al. (2009). "The effects of feeding rats diets deficient in folic acid and related methyl donors on the blood pressure and glucose tolerance of the offspring." Br J Nutr101(9): 1333-1340.

Maloney, C. A., S. M. Hay, et al. (2011). "A methyl-deficient diet fed to rat dams during the peri-conception period programs glucose homeostasis in adult male but not female offspring." $\underline{\mathrm{J} \text { Nutr} 141(1): ~ 95-100 .}$

McKay, J. A., L. Xie, et al. (2014). "Metabolic effects of a high-fat diet post-weaning after low maternal dietary folate during pregnancy and lactation." Mol Nutr Food Res58(5): 1087-1097. 
Miller, J. W., M. G. Garrod, et al. (2009). "Metabolic evidence of vitamin B-12 deficiency, including high homocysteine and methylmalonic acid and low holotranscobalamin, is more pronounced in older adults with elevated plasma folate." Am J Clin Nutr90(6): 1586-1592.

Mitchell, N. S., V. A. Catenacci, et al. (2011). "Obesity: overview of an epidemic." Psychiatr Clin North Am34(4): 717-732.

Morris, M. S., J. Selhub, et al. (2012). "Vitamin B-12 and folate status in relation to decline in scores on the mini-mental state examination in the framingham heart study." J Am Geriatr Soc60(8): 1457-1464.

Muthayya, S., A. V. Kurpad, et al. (2006). "Low maternal vitamin B12 status is associated with intrauterine growth retardation in urban South Indians." Eur J Clin Nutr60(6): 791-801.

Nicholas, L. M., J. L. Morrison, et al. (2016). "The early origins of obesity and insulin resistance: timing, programming and mechanisms." Int J Obes (Lond)40(2): 229-238.

O'Leary, F., M. Allman-Farinelli, et al. (2012). "Vitamin $\mathrm{B}(1)(2)$ status, cognitive decline and dementia: a systematic review of prospective cohort studies." Br J Nutr108(11): 1948-1961.

Reeves, P. G., F. H. Nielsen, et al. (1993). "AIN-93 purified diets for laboratory rodents: final report of the American Institute of Nutrition ad hoc writing committee on the reformulation of the AIN-76A rodent diet." J Nutr123(11): 1939-1951.

Refsum, H. and A. D. Smith (2008). "Are we ready for mandatory fortification with vitamin B-12?" Am J Clin Nutr88(2): 253-254.

Refsum, H., C. S. Yajnik, et al. (2001). "Hyperhomocysteinemia and elevated methylmalonic acid indicate a high prevalence of cobalamin deficiency in Asian Indians." Am J Clin Nutr74(2): 233-241.

Sabet, J. A., L. K. Park, et al. (2016). "Paternal B Vitamin Intake Is a Determinant of Growth, Hepatic Lipid Metabolism and Intestinal Tumor Volume in Female Apc1638N Mouse Offspring." PLoS One11(3): e0151579.

Selhub, J. and L. Paul (2011). "Folic acid fortification: why not vitamin B12 also?" Biofactors37(4): 269-271.

Selhub, J. and I. H. Rosenberg (2016). "Excessive folic acid intake and relation to adverse health outcome." Biochimie126: 71-78.

Shand, A. W., M. Walls, et al. (2016). "Dietary vitamin, mineral and herbal supplement use: a cross-sectional survey of before and during pregnancy use in Sydney, Australia." Aust N Z J Obstet Gynaecol56(2): 154-161. 
Sharma, D., S. Shastri, et al. (2016). "Intrauterine Growth Restriction: Antenatal and Postnatal Aspects." Clin Med Insights Pediatr10: 67-83.

Steegers-Theunissen, R. P., S. A. Obermann-Borst, et al. (2009). "Periconceptional maternal folic acid use of 400 microg per day is related to increased methylation of the IGF2 gene in the very young child." PLoS One4(11): e7845.

Steenweg-de Graaff, J., S. J. Roza, et al. (2015). "Associations of maternal folic acid supplementation and folate concentrations during pregnancy with foetal and child head growth: the Generation R Study." European Journal of Nutrition.

Stewart, C. P., P. Christian, et al. (2011). "Low maternal vitamin B-12 status is associated with offspring insulin resistance regardless of antenatal micronutrient supplementation in rural Nepal." J Nutr141(10): 1912-1917.

Suren, P., C. Roth, et al. (2013). "Association between maternal use of folic acid supplements and risk of autism spectrum disorders in children." JAMA309(6): 570-577.

Timmermans, S., V. W. Jaddoe, et al. (2009). "Periconception folic acid supplementation, fetal growth and the risks of low birth weight and preterm birth: the Generation R Study." Br J Nutr102(5): 777-785.

van Uitert, E. M., S. van Ginkel, et al. (2014). "An optimal periconception maternal folate status for embryonic size: the Rotterdam Predict study." BJOG121(7): 821-829.

Visentin, C. E., S. P. Masih, et al. (2016). "Low Serum Vitamin B-12 Concentrations Are Prevalent in a Cohort of Pregnant Canadian Women." J Nutr146(5): 1035-1042.

Wardlaw, T., A. Blanc, et al. (2004). United Nations Children's Fund and World Health Organization, Low Birthweight: Country, regional and global estimates. New York, UNICEF.

Whincup, P. H., S. J. Kaye, et al. (2008). "Birth weight and risk of type 2 diabetes: a systematic review." JAMA300(24): 2886-2897.

WHO (1995). "Physical status: the use and interpretation of anthropometry. Report of a WHO Expert Committee." World Health Organ Tech Rep Ser854: 1-452.

Williams, J., C. T. Mai, et al. (2015). Updated Estimates of Neural Tube Defects Prevented by Mandatory Folic Acid Fortification - United States, 1995-2011. Morbidity and Mortality Weekly Report, Centers for Disease Control and Prevention. 64: 1-5. 
Wolff, G. L., R. L. Kodell, et al. (1998). "Maternal epigenetics and methyl supplements affect agouti gene expression in Avy/a mice." Faseb J12(11): 949-957.

Wolff, G. L., D. W. Roberts, et al. (1986). "Prenatal determination of obesity, tumor susceptibility, and coat color pattern in viable yellow (Avy/a) mice. The yellow mouse syndrome." J Hered77(3): 151-158.

Yajnik, C. S., S. S. Deshpande, et al. (2008). "Vitamin B12 and folate concentrations during pregnancy and insulin resistance in the offspring: the Pune Maternal Nutrition Study." Diabetologia51(1): 29-38.

Yajnik, C. S., C. H. Fall, et al. (1995). "Fetal growth and glucose and insulin metabolism in four-year-old Indian children." Diabet Med12(4): 330-336. 